\title{
Tracking Influence Through Citation Index Comparisons and Preliminary Case Studies Panel Position Statement
}

\author{
Mary Ellen Zurko \\ IBM Software Group \\ 5 Technology Park Drive \\ Westford, MA 01886 USA \\ $+1-978-392-6018$ \\ mzurko@us.ibm.com
}

\begin{abstract}
We consider the influence of the New Security Paradigms Workshop by looking at the web citations to its papers in CiteSeer, and comparing those to another computer security workshop and a conference. We then go on to ask selected NSPW authors and NSPW 2001 attendees for their opinion of the influence of NSPW to date.
\end{abstract}

\section{General Terms}

Measurement, Security.

\section{Keywords}

Computer security research influence.

\section{INTRODUCTION}

One way to indicate the influence of a published paper is by its citations. Widely used citation indices, such as the Web of Science at MIT, only cover journals, not conference proceedings. They are of little value in tracking our workshop history, and of limited value in tracking computer security research, since much influential research is published in conference and workshop proceedings.

CiteSeer <http://citeseer.ni.nec.com> uses web searches to produce a citation index. Important computer security publications, such as the IEEE Security and Privacy conference proceedings, are not on the web. Thus, any citations to an NSPW paper from an S\&P paper do not show up in CiteSeer. However, the web is generally influential. I posit for the purposes of this draft position statement that the relative importance of a piece of research on the web reflects its relative importance in general. I further posit that its citation count in CiteSeer reflects its relative importance on the web.

Permission to make digital or hard copies of all or part of this work for personal or classroom use is granted without fee provided that copies are not made or distributed for profit or commercial advantage and that copies bear this notice and the full citation on the first page. To copy otherwise, or republish, to post on servers or to redistribute to lists, requires prior specific permission and/or a fee.

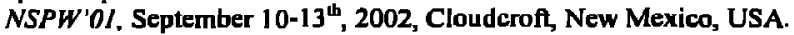

Copyright 2002 ACM 1-58113-457-6/01/0009...\$5.00.

\section{CITATION INDEX NUMBERS}

I did citations searches on CiteSeer on all papers published in the proceedings of the first nine years of NSPW. Below are some numbers of interest from those searches. It seems too carly to have numbers on NSPW 2000 (the only cite was a selfcite of a pre-print of one of the papers). So, the numbers only cover the first eight years of NSPW (1992 - 1999).

CiteSeer summarizes the number of citations to a paper in the form " $n(m)$ ", where $n$ is the number of citations it finds from papers whose set of authors do not overlap with the set of authors of the paper in question, and $m$ is the number of self citations it finds (papers where one or more authors are the same between the citing paper and the cited paper). This gives a total of $n+m$ unique citations found by CiteSeer.

Below is a list of NSPW papers with 2 or more external cites on CiteSeer. They are ranked by external citations, then self citations, then publication year:

Table 1: NSPW papers ranked by number of citations

15 (4) Rasmusson/Jansson's '96 paper "Simulated Social Control for Secure Ințernet Commerce”

10 (3) Erlingsson/Schneider's '99 paper "SASI Enforcement of Security Policies: A Retrospective"

7 (0) Bell's '94 paper "Modeling the "Multipolicy Machine""

6 (0) Zurko/Simon's '96 paper "User-Centered Security",

5 (1) Olawsky/Fine/Schneider/Spencer's 196 paper "Developing and Using a 'Policy Neutral' Access Control Policy"

5 (0) Wulf/Wang/Kienzle's '96 paper "A New Model of Security for Distributed Systems"

3 (4) Somayaji/Hofmeyr/Forrest's '97 paper

3 (0) Salter/Saydjari/Schneier/Wallner '98 paper

3 (0) Hosmer's '92 paper

3 (0) Strens/Dobson '93 paper

2 (0) Timmerman's '99 paper

2 (0) Riechmann/Hauck's '97 paper

2 (0) Abdul-Rahman/Hailes' 97 paper

2 (0) Timmerman's '97 paper 
2 (0) Blakely's '96 paper "The Emperor's Old Armor"

2 (0) Dobson's '92 paper

Below are the total CiteSeer citations I found for all NSPW proceedings, ranked similarly:

Table 2: NSPW years ranked by number of citations

34 (6) 196

13 (4) '99

12 (8) '97

7 (0) ' 94

6 (4) '98

$6(0) ' 92$

5 (2) 193

0 (3) '95

I now compare the NSPW 1996 numbers with the Computer Security Foundations Workshop (CSFW) and IEEE Symposium on Security and Privacy (S\&P) from the same year.

Table 3: Comparison of CiteSeer ciltations to 1996 NSPW, CSFW, and SerP

\begin{tabular}{|l|l|l|l|}
\hline & NSPW & CSFW & S\&P \\
\hline Total citations & $34(6)$ & $88(24)$ & $393(72)$ \\
\hline Total papers & 17 & 17 & 23 \\
\hline NSPW compare & -- & $37 \%(25 \%)$ & $9 \%(9 \%)$ \\
\hline Normalized number & $34(6)$ & $88(24)$ & $290(53)$ \\
\hline $\begin{array}{l}\text { Normalized } \\
\text { compare }\end{array}$ & -- & $37 \%(25 \%)$ & $12 \%(11 \%)$ \\
\hline Top 2 papers cites & $21(4)$ & $32(5)$ & $207(21)$ \\
\hline Top 2 papers \% & $66 \%(66 \%)$ & $36 \%(21 \%)$ & $53 \%(29 \%)$ \\
\hline
\end{tabular}

Table 3 shows that 1996 NSPW has approximately 1/3 the external CiteSeer citations of 1996 CSFW and approximately $1 / 10$ the external CiteSeer citations of 1996 S\&P. NSPW has approximately the self citations of CSFW, and approximately $1 / \overline{10}$ the self citations of S\&P. The normalized S\&P numbers reflect the fact that that NSPW has $74 \%$ of the papers that S\&P has.

As I ran the numbers, it seemed to me that the most heavily cited papers strongly influenced any particular year's proceedings. The last two rows in Table 3 shows the total number of the citations to the r,op two papers in each proceedings, and the percentage of the citations of that year's proceedings that are attributed to those two most heavily cited papers (unnormalized). In particular, those papers are:

NSPW:

- "Simulated Social Control for Siecure Internet Commerce", Lars Rasmusson, Sverker Jansson [15 (4)]
- "User-Centered Security", Mary Ellen Zurko, Richard T. Simon [6 (0)]

CSFW:

- "Some New Attacks upon Security Protocols", Gavin Lowe [20 (2)]

- "Intensional specification of security protocols", A. W. Roscoe [12 (3)]

S\&P:

- "Decentralized Trust Management", Matt Blaze, Joan Feigenbaum, Jack Lacy. [113 (17)]

- "Java Security: From HotJava to Netscape and Beyond", Drew Dean, Edward W. Felten, Dan S. Wallach [94 (4)]

\section{PRELIMINARY CASE STUDIES}

I could find nothing in the CiteSeer numbers to indicate that NSPW had been particularly influential in its first 8 years. I then considered that NSPW's influence was not reflected in the numbers. One way a new paradigm paper can influence computer security research is if it catches researchers' imaginations, is widely read and debated, and influences the course of future research. "Decentralized Trust Management" is, to my mind, a classic example of that model, and the numbers bear it out. However, a new paradigm paper's influence can be less direct. It can influence specific work or particular researchers, which then go on to have a larget, more obvious, and more quantifiable impact on computer security. Determining any influence like that would require doing citation index hierarchy look ups, and understanding in some detail which citations were influential, and which were merely filler for the Related Work section of a paper.

That level of research is out of scope for the current position paper. Instead, I sent email to the authors of the top 6 papers in Table 1, as well as the author of an NSPW paper that I thought was highly influential but did not have 5 or more CiteSeer citations. I sent a brief description of the panel, a CiteSeer link to the citations I found on their work, a short description of anything interesting $I$ found in their citations, and an invitation for comment on the influence their paper had. 4 of the 7 responded.

The CiteSeer citations I had found on "Simulated Social Control for Secure Internet Commerce" included 5 from authors that I recognized from NSPW. Their work seemed to have been most cited in the agent context, particularly around agent security and marketplace issues. Sverker Jansson replied to my query. The paper was based on his co-author's MSc project. They did not continue that particular line of research. "There wasn't much of a community with these interests at the time, not enough theory for us to build on, and we weren't in a position to create a new foundation." Sverker and Lars do most of their work on agents. They announced their papers on agent mailing lists, and published a paper on the same topic in the PAAM agent conference. Lars has moved into the field of automation of electronic markets by means of methods from mathematical finance. "This is in some sense a related field, as we were looking for economic models for quantifying trust."

The CiteSeer citations to "SASI Enforcement of Security Policies: A Retrospecitive" seemed to be mostly about language security. All the self references it found were from 
Schneider. Fred Schneider responded to my query. Their NSPW paper was the first detailed explanation of In-lined Reference Monitors (IRM) and the first report of a prototype. The approach itself was first mentioned in Schneider's earlier ACM TOIS paper on Enforceable Security Policies, and documented again in an S\&P paper directly after NSPW. Schneider says it is clearly too early to say whether the work will have an impact. "There is all sorts of circumstantial evidence that the work is attracting interest. I'm just back from giving a keynote lecture on our work in Paris at the Static Analysis Symposium; and I'm typing this email while in a Redmond hotel--uSoft is collaborating on a NET port, since IRMs seem like a promising way to address many of their problems with hostile mobile code." Schneider thinks "the trend to note is the advent of 'language based security', a new collection of policy enforcement techniques based on programming language technology (program re-writing and program analysis). IRM and PCC (proof carrying code) are examples (and, perhaps not by accident, are complimentary approaches that actually leverage each other). [The way to view PCC is as a generalpurpose means for relocating components in a trusted computing base.] Our IRM NSPW citation count could be indicative of the role this paper serves as an early effort in this new subfield."

I was surprised that "The Emperor's Old Armor" didn't come up with more citations from CiteSeer. It had created a lot of interest that year, at NSPW, at the NSPW panel at NISSC, and at the followon panel at S\&P. Bob Blakeley replied to my query. "The paper is cited a lot in print (I don't keep a list, but I see it regularly and imagine it's up in the scores of references). Has it had an influence? I think it's hard to tell. I always thought the older generation would have to retire before we'd dig out of this rut; maybe mine will too.... On the other hand, if I'm right and the problem really is insoluble using the current model, then people will find alternative models in the course of time anyway (as they're clearly trying to do in some areas) whether they've been influenced by the paper or not. $A$ conundrum." He also speculated that one reason the paper is not cited more is that he doesn't have a web site so it isn't freely available electronically anywhere.

The CiteSeer database is missing references to "User-Centered Security" from both S\&P (at least one self-cite) and Usenix Security Symposium. Wondering about CiteSeer's web coverage, I did a quick search on Google for both "UserCentered Security" and "Emperor's Old Armor". The first few pages of both turned up references that seemed like they should be covered in CiteSeer, but aren't. Our "User-Centered Security" paper at NSPW was meant to try to pull together the research available to date in that area, and to inspire further work. Since then, a community has sprung up. There is an online bibliography in Human Factors and Computer Security htto://www_sims,berkeley.edu/-alma/biblio.html and a distribution list for discussions on the topic. I do believe it provided a useful one stop bibliography for researchers interested in that area for the first few years after its publication. It also named and helped legitimize the field of work. When I published that paper, most people laughed at the notion of putting the two disciplines together.

\section{DISCUSSION}

A number of issues were raised during discussion of my findings from CiteSeer and selected NSPW authors. Carol Taylor suggested that there might be other measures of influence, that might best be brought out with a survey of past NSPW participants. John McHugh suggested the more obviously scholarly approach of searching the literature for subfields that were initiated at or substantially furthered by NSPW, such as the inline reference monitor and immune system approaches to intrusion detection.

Ken Olthoff suggests measuring depth of influence on attendees over breadth of influence on others, which is also more in line with articulated NSPW goals. Marv Schaefer pointed out that at NSPW most of the insights and good discussions come from the sessions, while at other conferences and workshops they occur during the breaks.

Victor Raskin said that citations often come through having PhD students' papers that generate a lot of references, and not many attendees have graduate students. He has also heard that universities are considering dropping citation indices as a criterion for promotions because the data is worthless, which calls into question the idea of using them to track influence of a workshop or conference.

\section{CONCLUSIONS}

CiteScer numbers do not indicate that past NSPW proceedings have been particularly influential on the web. Preliminary discussions with the authors of some of the most highly cited papers from NSPW do not form a clear picture of NSPW's contribution to the work that followed from their NSPW papers. Discussion at the workshop suggests that there might be other, most useful measurement of influence. We might also look at the depth vs. breadth of influence. There is some suggestion that citation indices are being discredited as easy tools to measure influence.

\section{ACKNOWLEDGMENTS}

Special thanks to the authors from past years who responded to my email survey. Many thanks to the NSPW participants, and for the lively discussion and ideas as sketched in the discussion section. 